\title{
Research of the Affection of "Plate Feather Shuttlecock" on Physical Qualities of the Middle School Students
}

\author{
SUN Ding-fu ${ }^{1}$, Luo-ling ${ }^{1}$, Lu-yan ${ }^{2}$ \\ ${ }^{1)}$ Physical Education Dept, Qiannan Normal University of Nationalities, Duyun, Guizhou, China; \\ ${ }^{2)}$ No.3 Middle School of Fuquan in Guizhou, Fuquan, Guizhou, China.
}

\begin{abstract}
In order to improve the system of research in the plate feather shuttlecock, discuss the influence on middle school students' physical qualities, forty-five students from grade seven in the No.3 Middle School of Fuquan in Qiannan State were invited to participate in the 15 weeks' study. The result of the research indicates that students' physical quality have been improved a lot in many aspects. Such as, in spanning over and over again, in on balance of wood, in Autochthonous vertical jump and in Back force and Grip strength; It also shows that long-term plate feather shuttlecock practice can greatly improve the students' sensitive quality and physical balance and muscle strength. In conclusion, the plate feather shuttlecock is the best choice in school-based teaching material in the minority region.
\end{abstract}

Keywords - Plate feather shuttlecock, School-based teaching material, Middle School Students, physical quality

\section{板羽建对中学生运动素质的影响研究}

\author{
孙定福 $^{1} \quad$ 罗玲 ${ }^{1} \quad$ 陆艳 $^{2}$ \\ 1) 黔南民族师范学院体育系，都匀，贵州，中国 \\ 2) 贵州省福泉市第三中学, 福泉, 贵州, 中国
}

\begin{abstract}
摘 要 为完善板羽建的研究体系、讨论板羽建对中学生运动素质的影响, 特以黔南州福泉市第三中学七年级 45 名同学为研究对 象, 进行了为期 15 周的教学实验研究。研究结果显示: 实验后学生的反复横跨、踩木平衡、原地纵跳、背力和握力指标均有提高, 各 项成绩均具有显著性差异。实验证明长期进行板羽毽练习能有效提高学生的灵敏素质、平衡能力及肌肉力量等素质, 是少数民族地区 学校体育校本教材建设中较为科学合理的首选素材。
\end{abstract}

关键词 板羽珴, 校本教材, 中学生, 运动素质

\section{1. 问题的提出}

教材建设是布依族地区学校体育课程改革的关键环 节, 是校本课程建设的重点。为使布依族地区学校体育课 程资源的开发与利用更趋于科学化, 教育部人文社会科学 研究西部与边疆地区项目《布依族地区学校体育课程资源 的开发性研究》课题组, 特将黔南布依族苗族地区的民族 民间传统体育项目“板羽珴”引进黔南州福泉市第三中学进 行教学实验, 开展教学研究工作。

基金项目: 教育部人文社会科学研究西部和边疆地区项目 (10XJA880002)。
板羽稉是黔南苗族同胞用一块木板制作成简易的板羽 拍, 用羽毛和小竹管制作成建子, 通过手持木板, 击打建 子，使建子在空中来回飞舞的一项民族民间体育项目。1939 年, 中华全国体育协进会干事魏振武、李国堂等到贵州都 匀一带考察时, 看见苗族青年进行“打建”的游戏, 他们受 到启发后综合羽毛球和网球的部分规则, 编写了板羽的建的 比赛规则和比赛方法, 经中华全国体育协进会总干事董守 义先生审校, 正式将这项运动定名为“板羽球”[1]（黔南苗 族同胞习惯称 “板羽建”)。由此, 使得此项运动在全国得以 传扬和发展。

板羽稉因制作简单, 动作易学, 对场地、器材的要求 
不高, 并具有地域性民族特色, 因此, 在课程资源开发性 研究过程中, 被列为黔南州福泉市第三中学的体育与健康 校本教材开发项目。为保证开发项目的科学性与健身价值, 项目组特安排为期 15 周的教学实验, 探讨板羽建对中学生 运动素质的影响, 旨在为板羽的建的健身价值提供实验支撑, 为布依族地区中学体育校本教材建设提供理论依据。

\section{2. 实验对象与方法}

\section{1 实验对象}

根据福泉三中体育校本课程建设和教材教学实验安 排, 选取七年级四班 45 名学生为研究对象, 其中女生 24 人, 平均年龄 12.4 岁, 身高 $149.29 \pm 6.36$ 厘米, 体重 $43.08 \pm 7.2$ 千克; 男生 21 人, 平均年龄 12.7 岁, 身高 $152.19 \pm 6.79$ 厘 米, 体重 $44.37 \pm 6.35$ 千克。每位实验对象均无运动障碍和 疾病史, 符合教学实验要求。

\section{2 实验方法}

\subsection{1 干预方案}

实验班围绕板羽的建技术动作和比赛规则进行 15 周的 教学实验, 每周 2 次课, 每次 45 分钟, 教学内容主要选择 发球技术、接发球技术、跑动技术、双打技术和教学比赛 等技、战术动作, 每次教学按 Table I 的方案具体执行。

TABLE I The program of experimental intervention

\begin{tabular}{|c|c|c|c|c|}
\hline 教学阶段 & $\begin{array}{c}\text { 时间 } \\
\text { /min }\end{array}$ & $\begin{array}{c}\text { 干预方法与 } \\
\text { 训练内容 }\end{array}$ & 形式与练习 & 备 注 \\
\hline 热身 & $5-10$ & $\begin{array}{c}\text { 热身活动和 } \\
\text { 全身运动 }\end{array}$ & 集体练习 & $\begin{array}{c}\text { 身体微微 } \\
\text { 出汗 }\end{array}$ \\
\hline 学习体验 & $30-35$ & $\begin{array}{l}\text { 板羽珴技术动 } \\
\text { 作和教学比赛 }\end{array}$ & $\begin{array}{c}\text { 分组练习和 } \\
\text { 分场地比赛 }\end{array}$ & $\begin{array}{c}\text { 复习之前所学 } \\
\text { 内容和新教 } \\
1-2 \text { 个技术内容 }\end{array}$ \\
\hline 恢复身心 & $3-5$ & $\begin{array}{c}\text { 放松练习 (主 } \\
\text { 要是臂和腿部 } \\
\text { 肌肉的放松) }\end{array}$ & $\begin{array}{c}\text { 集体模仿教 } \\
\text { 人相互放松 }\end{array}$ & $\begin{array}{c}\text { 可配合音乐 } \\
\text { 进行身心放松 }\end{array}$ \\
\hline
\end{tabular}

\subsection{2 实验指标与测试方法}

根据板羽珴运动特点、场地器材和人力条件, 参考高 等教育出版社 1990 年 9 月出版的《人体测量与评价》中相 关指标的测试方法, 并通过专家评估、审定“反复横跨”、“踩 木平衡”、“原地纵跳”、“背力”和“握力”五项指标作为评定 实验对象灵敏、平衡及力量素质的实验指标。各指标测试 方法与要求如下:

\section{(1)反复横跨}

根据《人体测量与评价》一书中的测试方法进行, 记 录 20 次循环侧跨所用的时间[2]。要求测试时只许横向跨步 移动, 且两脚必须跨在线的两侧, 违例的需重新进行测试。

\section{(2)原地纵跳}

根据《人体测量与评价》一书中的测试方法, 连续进行 3 次试跳, 取最佳成绩记录[2]。测试时要求起跳和落地均 用双脚, 不得跨步、垫步, 手臂可做预摆动作。原地伸臂 点指印时, 臂要充分伸直, 体侧要轻贴墙壁。

(3)踩木平衡

受试者以优势脚全脚平行纵向踩在长 30 厘米、高、宽 各 3 厘米的方木条上, 支撑腿伸直, 另一腿屈膝抬起脚离 地面 $30 \mathrm{~cm}$ 。同时两臂侧举, 计算单脚站在木条上维持身体 平衡的时间。每次记录从单脚踩上木条另一脚离地 $30 \mathrm{~cm}$ 开始, 到任一脚触接地面或两臂晃动偏离水平面 15 度为止 的时间。每人测试两次, 取平均成绩。受试者应充分活动 踝关节, 谨防受伤。

\section{(4)背力}

采用常州市钱璟康复器材有限公司生产的钱璟牌 F-BLJ 电子背力计进行标准化测试。每位受试者连测 2 次, 记录最好成绩。

\section{(5)握力}

采用常州市钱璟康复器材有限公司生产的钱璟牌 F-MLJ 电子握力计进行标准化测试。每位受试者用优势手 连续进行 2 次测试, 记录最佳成绩。

\section{2 .3 实验论证}

运用 Excel2003 和 SPSS17.0 统计软件对实验前、后测 试数据进行配对样本 $\mathrm{T}$ 检验, 探讨板羽稉对中学生运动素 质的影响是否具有显著性。比较参数用 $\bar{x} \pm \mathrm{s}$ 表示, 显著水 平为: $\mathrm{P}>0.05$ (无显著性差异); $\mathrm{P}<0.05$ (具有显著性差异); $\mathrm{P}<0.01$ (具有高度显著性差异)。

\section{3. 实验结果与分析}

\section{1 实验结果}

\section{1 .1 受试者反复横跨结果}

实验前, 测得男生反复横跨成绩为 $13.31 \pm 1.67$ 秒, 女 生成绩 $13.72 \pm 2.13$ 秒。通过一个学期的板羽建教学实验后, 再次测得男生成绩为 $12.35 \pm 1.53$ 秒, 女生成绩为 $12.89 \pm 2.01$ 秒, 男、女生成绩分别比实验前提高了 0.96 秒和 0.83 秒, 成绩提高幅度分别为 $7.21 \%$ 和 $6.05 \%$ 。通过采用“两配对样 
本 $\mathrm{t}$ 检验”后得出, 该项测试男、女生实验前、后成绩均具 有高度显著性差异，P<0.01（见 Table II）。

TABLE II Experimental results and statistics of the Spanning Over and Over again

\begin{tabular}{|c|c|c|c|c|c|}
\hline & 实验前 $(\mathrm{s})$ & 实验后 $(\mathrm{s})$ & 增量 $(\mathrm{s})$ & 增幅 $(\%)$ & $\mathrm{P}$ \\
\hline $\begin{array}{c}\text { 男生 } \\
\mathrm{N}=21\end{array}$ & $13.31 \pm 1.67$ & $12.35 \pm 1.53$ & -0.96 & -7.21 & 0.000 \\
\hline $\begin{array}{c}\text { 女生 } \\
\mathrm{N}=24\end{array}$ & $13.72 \pm 2.13$ & $12.89 \pm 2.01$ & -0.83 & -6.05 & 0.000 \\
\hline
\end{tabular}

实验证明, 经常参加板羽建运动能提高学生的运动能 力，增强机体的灵敏与协调性。

\section{1 .2 受试者踩木平衡结果}

实验前, 测得男生踩木平衡成绩为 $50.96 \pm 12.4$ 秒, 女 生成绩 $47.48 \pm 11.37$ 秒。通过一个学期的板羽建教学实验 后, 再次测得男生踩木平衡成绩为 $56.58 \pm 10.82$ 秒, 女生成 绩为 $51.74 \pm 10.42$ 秒, 男、女生成绩分别比实验前提高了 5.62 秒和 4.26 秒，成绩提高幅度分别为 $11.03 \%$ 和 $8.97 \%$ 。 通过采用“两配对样本 $\mathrm{t}$ 检验”后得出, 男、女生该项成绩实 验前、后具有显著性差异， P $<0.05$ (见 TableIII)。

TABLE III Experimental results and statistics of the On Balance of Wood

\begin{tabular}{|c|c|c|c|c|c|}
\hline & 实验前 $(\mathrm{s})$ & 实验后 $(\mathrm{s})$ & 增量 $(\mathrm{s})$ & 增幅 $(\%)$ & $\mathrm{P}$ \\
\hline $\begin{array}{c}\text { 男生 } \\
\mathrm{N}=21\end{array}$ & $50.96 \pm 12.4$ & $56.58 \pm 10.82$ & 5.62 & 11.03 & 0.013 \\
\hline $\begin{array}{c}\text { 女生 } \\
\mathrm{N}=24\end{array}$ & $47.48 \pm 11.37$ & $51.74 \pm 10.42$ & 4.26 & 8.97 & 0.035 \\
\hline
\end{tabular}

实验证明, 经常从事板羽建运动能有效提高练习者的 平衡能力。

\section{1 .3 受试者原地纵跳结果}

实验前, 实验对象男、女同学原地纵跳成绩分别为 $29.41 \pm 3.17$ 厘米和 $24.18 \pm 2.7$ 厘米, 经过 15 周的板羽珴教 学训练后, 再次测得男生成绩为 $31.89 \pm 4.11$ 厘米, 女生成 绩为 $27.46 \pm 4.82$ 厘米, 男、女同学该项成绩分别提高了 2.48 厘米和 3.28 厘米, 成绩提高幅度分别为 $8.43 \%$ 和 $13.56 \%$ 。 经统计分析得出, 男、女生实验前、后原地纵跳成绩均具 有显著性差异， $\mathrm{P}<0.05$ (见 TableIV)。

实验证明, 板羽珴练习能有效地增强中学生的下肢弹 跳能力。
TABLE IV Experimental results and statistics of the Autochthonous Vertical Jump

\begin{tabular}{|c|c|c|c|c|c|}
\hline & 实验前 $(\mathrm{cm})$ & 实验后 $(\mathrm{cm})$ & 增量 $(\mathrm{cm})$ & 增幅 $(\%)$ & $\mathrm{P}$ \\
\hline $\begin{array}{c}\text { 男生 } \\
\mathrm{N}=21\end{array}$ & $29.41 \pm 3.17$ & $31.89 \pm 4.11$ & 2.48 & 8.43 & 0.008 \\
\hline $\begin{array}{c}\text { 女生 } \\
\mathrm{N}=24\end{array}$ & $24.18 \pm 2.70$ & $27.46 \pm 4.82$ & 3.28 & 13.56 & 0.001 \\
\hline
\end{tabular}

\subsection{4 受试者背力结果}

实验前, 测得实验对象男、女同学背力成绩分别为 $58.71 \pm 8.67$ 千克和 $45.44 \pm 7.45$ 千克, 经过一个学期的板羽 建教学实验后, 测得男生成绩为 $61.52 \pm 6.17$ 千克, 女生成 绩为 $48.09 \pm 6.04$ 千克, 男、女同学成绩比实验前分别提高 了 4.79 千克和 5.83 千克, 成绩提高幅度分别为 $4.79 \%$ 和 $5.83 \%$ 。通过采用“两配对样本 $\mathrm{t}$ 检验”后得出, 男、女同学 该项测试实验前、后成绩差异具有显著性， P $<0.05$ （见 Table V)。

TABLE V Experimental results and statistics of the Back Force

\begin{tabular}{|c|c|c|c|c|c|}
\hline & 实验前 $(\mathrm{kg})$ & 实验后 $(\mathrm{kg})$ & 增量 $(\mathrm{kg})$ & 增幅 $(\%)$ & $\mathrm{P}$ \\
\hline $\begin{array}{c}\text { 男生 } \\
\mathrm{N}=21\end{array}$ & $58.71 \pm 8.67$ & $61.52 \pm 6.17$ & 2.81. & 4.79 & 0.012 \\
\hline $\begin{array}{c}\text { 女生 } \\
\mathrm{N}=24\end{array}$ & $45.44 \pm 7.45$ & $48.09 \pm 6.04$ & 2.65 & 5.83 & 0.003 \\
\hline
\end{tabular}

实验证明，长期进行板羽稉练习或比赛能有效地增强 中学生的背部力量。

\section{1 .5 受试者握力结果与分析}

实验前, 测得实验对象男、女同学握力成绩分别为 $34.22 \pm 4.50$ 千克和 $29.83 \pm 6.56$ 千克, 经过一个学期的板羽 建教学实验后, 再次测得男生成绩为 $35.28 \pm 3.55$ 千克, 女 生成绩为 31.04 \pm 5.61 千克, 男、女同学实验后成绩比实验 前分别提高了 1.06 千克和 1.21 千克, 成绩提高幅度分别为 $3.10 \%$ 和 4.06\%。通过采用“两配对样本 $\mathrm{t}$ 检验”后得出, 男、 女同学该项测试实验前、后成绩具有显著性差异, $\mathrm{P}<0.05$ (见 TableVI)。

TABLE VI Experimental results and statistics of the Grip Strength

\begin{tabular}{|c|c|c|c|c|c|}
\hline & 实验前(s) & 实验后(s) & 增量 $(\mathrm{s})$ & 增幅 $(\%)$ & $\mathrm{P}$ \\
\hline $\begin{array}{c}\text { 男生 } \\
\mathrm{N}=21\end{array}$ & $34.22+4.50$ & $35.28 \pm 3.55$ & 1.06 & 3.10 & 0.032 \\
\hline $\begin{array}{c}\text { 女生 } \\
\mathrm{N}=24\end{array}$ & $29.83 \pm 6.56$ & $31.04 \pm 5.61$ & 1.21 & 4.06 & 0.018 \\
\hline
\end{tabular}

实验证明, 长期进行板羽珴练习或比赛能有效地增强 中学生的手臂力量。 


\section{2 实验分析}

3.2.1 板羽稉对机体灵敏素质的提高具有积极的作用

灵敏是指运动员迅速改变体位、转换动作和随机应变 的能力[3]。反复横跨是测试灵敏素质较为经济实效的指标。 测试中要求受试者在三条平行线间快速来回跨步移动, 以 横跨一定次数的用时长短来评定成绩, 用时越短, 说明灵 敏素质越好。

板羽的建的运动, 要求练习者根据来球情况, 不断在场 中前后或左右快速移动, 以最快的身体变位来接球反击, 达到夺分取胜的目的。实验班长期参加板羽建运动, 反复 横跨的成绩比实验前提高了, 说明实验对象反复改变体位、 随机调整身体重心的速度提高了, 即反应能力得到了提高, 从而提高了练习者的灵敏素质。由此可见, 板羽建对机体 灵敏素质的提高具有积极的作用。

\subsection{2 板羽珴能有效改善机体的平衡能力}

平衡是指身体所处的一种姿态以及在运动或受到外力 作用时能够自动调整并维持姿势的能力, 可分为静态平衡 和动态平衡, 其中静态平衡能力是指人体在相对静止的状 态下, 维持身体某种特定姿势一段时间的能力[3]。根据可 行条件, 本研究选取踩木平衡来评价实验对象的静态平衡 能力。

平衡成绩的好坏主要取决于机体的视觉反应速度、前 庭器的功能性质、本体感受器的功能状态和神经中枢的协 调整合功能[4]。板羽珴运动要求练习者根据空中建球的方 向、高度和速度来迅速调整自身姿态来挥拍击打, 这一练 习是在视觉的作用下, 通过神经系统协调全身各关节与肌 肉本体感受器, 从而保证技术动力发力时的身体平衡。实 验对象踩木平衡成绩提高了, 说明板羽建运动对维持机体 平衡有关的视觉、前庭觉、本体感觉和神经系统均具有积 极的影响作用, 因而机体的平衡能力得到有效的改善。

\subsection{3 板羽建能全面发展机体的力量素质}

力量是指神经肌肉系统在工作时克服或对抗阻力的能 力 [5], 是其它运动素质的基础, 是身体训练水平中最重要 的评定指标。原地纵跳是评定下肢力量的重要指标, 它是 通过屈趾蹬地、伸膝、展骼, 配合摆臂动作带动身体重心 向上垂直腾起的技术动作, 主要反映踝、膝关节的屈伸力 量和全身的协调能力。背力是评定躯干力量的重要指标, 它是在腿、臂协调支持下, 通过斜方肌、背阔肌等背部肌 肉收缩所表现出来的力量。握力是评定上肢力量的重要指 标, 它是通过五指内屈所表现出来的力量。

板羽珴运动, 常常要求练习者快速蹬地跑动或向上跳,
通过手持球拍协调下肢与躯干用力接、挡或扣杀键子, 这 样的练习使练习者躯干及四肢肌肉得到有效的锻炼, 使膝、 踝和肩关节的稳固性得到提高, 有效地增强了全身骨骼肌 和肌肉神经系统的协调性, 从而有效提高板羽建练习者的 力量素质。实验后实验对象原地纵跳成绩、背力成绩和握 力成绩均得到提高, 充分说明了板羽建能全面地发展机体 的力量素质。

\section{4. 结论与建议}

\section{1 结论}

4.1.1 经过 15 周的板羽珴练习或比赛, 能有效提高练习者 的灵敏、平衡及力量等身体素质, 特别是对灵敏素质和背 部力量素质的提高具有非常显著的作用。

4.1.2 板羽建技术动作和比赛规则类似于羽毛球运动, 能够 全面、系统地提高练习者的运动能力及健康水平。

4.1.3 板羽建因器材制作简易, 动作技术简单, 又是黔南布 依族苗族地区学生喜闻乐见的民族民间传统体育项目, 因 此, 是布依族地区中学体育校本课程教学内容的首选项目。

\section{2 建议}

4.2.1 板羽建是布依族苗族地区学校体育校本教材的首选 素材。板羽毽对中学生运动素质的提高具有积极的作用, 对运动场地和器材又无严格要求, 并且制作方法简单, 适 合引进体育课堂, 是少数民族地区学校体育校本课程资源 开发的好项目, 即能解决场地器材及办学经费的不足, 又 能满足少数民族地区学校、教师和学生的需求, 适合引进 体育与健康教学。

4.2.2 西部少数民族地区学校根据自身学校的具体情况, 针 对板羽建简单易学的技术, 灵活可变的规则和对身体素质 有效提高的特点, 将其素材教材化之后合理引进体育课堂。

\section{参考文献}

[1] Xu-Ye, Liu-liguo. Development of Miao Chicken Feather huttlecock. J. Sports Culture Guide, 2nd, pp. 89-93, 2011.

[2] The group of compile RENTI CELIANG YU PINGJIA. RENTI CELIANG YU PINGJIA. Beijing: Higher Education Press, September 1990.

[3] Deng-Shuxun, Wang-jian, Qiao-Decai. YUNDONG SHENGLIXUE. Beijing: Higher Education Press, 2007, pp.218.

[4] Liu-Yang, Development of Balance Measurement and Training Studies for Human Beings. J. Journal of Shenyang Sport University, vol.26, no.4, pp. 75-77, 2007.

[5] Tian-ye. YUNDONG SHENGLIXUE GAOJI JIAOCHENG. Beijing: Higher Education Press, 2003, pp. 31. 